\title{
Extra skeletal osteosarcoma suprascapular region: A case report
}

\author{
Badaruddin Sahito ${ }^{1}$, Soughat Katto ${ }^{2}$, Fareeha Masood ${ }^{3}$, Gul e Zehra ${ }^{3}$, Afshan Salah Uddin ${ }^{3}$, \\ Nasir Ud Din ${ }^{4}$ \\ 1 FCPS, Department of Orthopedic Surgery, Dr Ruth KM Pfau Civil Hospital, Karachi, Pakistan \\ 2 MS, Department of Orthopedic Surgery, Dr Ruth KM Pfau Civil Hospital, Karachi, Pakistan \\ 3 th Year MBBS, Dow Medical College, Karachi, Pakistan \\ 4 MBBS, FCPS, Department of Pathology and Laboratory Medicine, The Aga Khan University, Stadium Road, Karachi, Pakistan \\ *Corresponding Author: Gul e Zehra, 4th Year MBBS, Dow Medical College, Karachi, Pakistan. \\ Email: gulezehra6@gmail.com
}

Received: November 25, 2021; Accepted: December 23, 2021

\section{Abstract}

Extra skeletal osteosarcoma is a high-grade malignant tumor of an osteoid and cartilaginous matrix arising in the soft tissue without connection to the bone. It is known clinically for its aggressive behavior, poor prognosis, frequent recurrence and its resistance to chemotherapy, calling for expert diagnosis and necessary treatment measures. To the best of our knowledge, it is extremely rare comprising of $2-5 \%$ of all osteosarcomas and less than $1 \%$ of soft tissue tumors. We report a case of a 63 -year-old with an isolated lump in right supra scapular region. After imaging, surgical excision and pathological analysis, the diagnosis of extra skeletal osteosarcoma was made. This report documents the clinical and pathological features of extra skeletal osteosarcoma in this case.

Keywords: Osteosarcoma, Extraskeletal, Osteoid, Malignant.

\section{INTRODUCTION}

Osteosarcoma is a common malignant bone tumor of mesenchymal origin commonly found in the metapyseal region of long bones e.g., femur and tibia [1]. Extra skeletal osteosarcoma comprises of $2-5 \%$ of all osteosarcomas and $1 \%$ of all soft tissue tumors generally manifested in elderly individuals of age 40-70 years with a slight predisposition towards male [2] [3]. It is an infrequent malignant presentation of soft tissue occurring in the vicinity of bone comprising of connective tissue, bone or cartilage material with the risk linked to previously performed radiotherapy for other cancers [3]. Most of the tumor is hidden deep within the tissue though a few have been found beneath the skin too. Common site of presentation is thigh followed by buttocks, shoulder and trunk [3]. The tumor tends to show up with tenderness and swelling [4]. Compared to the osteosarcoma, it has poor prognosis due to its aggressive nature. Wide surgical resection followed by chemotherapy is the standard mode of treatment [3]. An informed consent was taken from the patient to publish this case report.

\section{CASE REPORT}

We present a case of a 63-year male who reported at the department of Orthopedics Surgery, Dow University of Health Sciences/ D Ruth K M Pfau Civil hospital Karachi in 2018 with a lump over the right supra scapular region for 1 year, that initially was small in size and increased slowly. Lump was associated with mild aching discomfort but not associated with fever and weight loss. A general surgeon operated him 2 months earlier he presented to our department. Biopsy showed extra-skeletal osteosarcoma. Three cycles of Neoadjuvant chemotherapy were done and the patient was referred to our outpatient department for further treatment. He was hypertensive and took medications for it.

On examination, there was a healed scar of about $6 \mathrm{~cm}$ at the suprascapular region with puckered skin in center. Swelling was of $12 \times 9 \mathrm{~cm}$ in size, firm to hard in consistency, adhered to underlying structure and free from skin. Shoulder and cervical spine movement were normal. Distal neurovascular status was intact in right upper limb. Hematological investigations were normal. X-ray scapular region was normal. Magnetic resonance imaging with gadolinium enhanced contrast showed hyper intense lesion with $9 \times 8 \mathrm{~cm}$ suprascapular area involving 
supraspinatus and levator scapula.

CT scan chest was free of any metastasis. Bone scan was negative for any bony mets. Case was discussed at musculoskeletal tumor board meeting and planned for wide margin resection.

Surgical Procedure: After consent and counseling, patient was operated under general anesthesia in prone position. Elliptical incision was made (Figure 1).

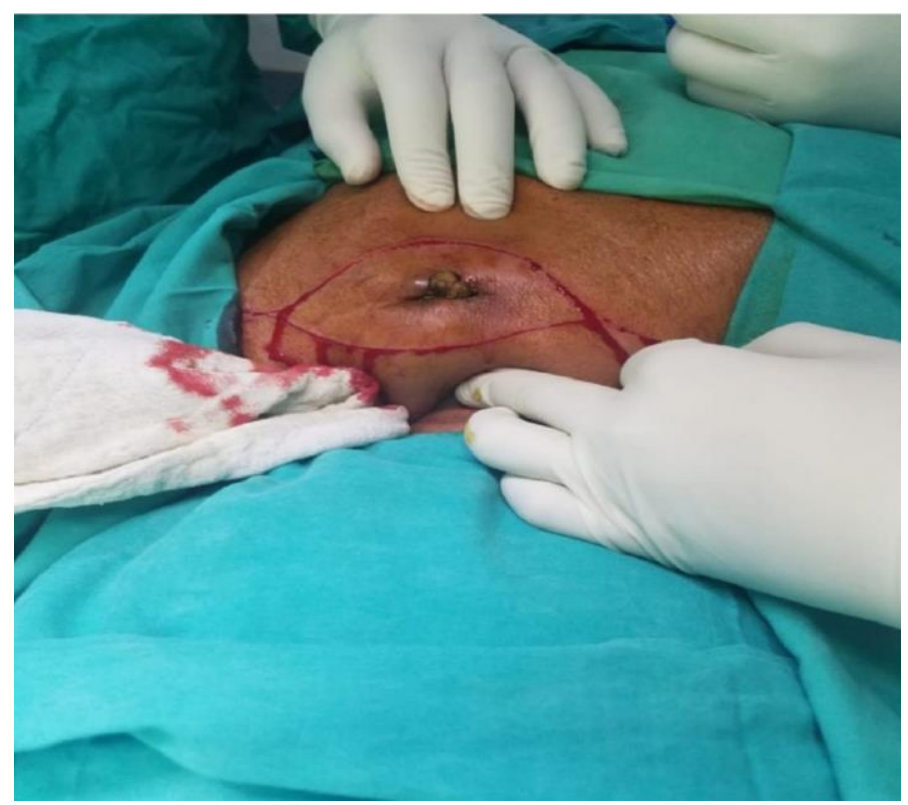

Figure 1: Incision mark

Flap raised and tumor was dissected free from surrounding muscle and then excised. Wound was closed in layers. Specimen was sent for histopathology. (Figure 2) Stitches were removed at two weeks.

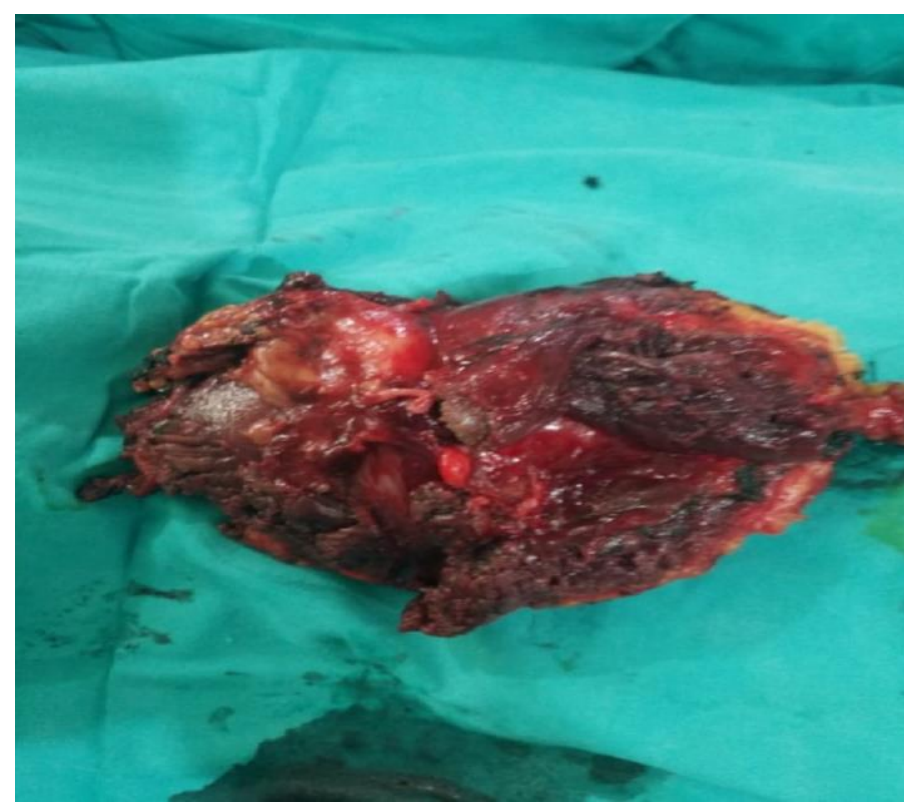

Figure 2: Resection specimen
Histopathology report showed that Gross examination specimen had ellipsed skin covered with fibro muscular tissue of $11.5 \times 8.5 \times 4 \mathrm{~cm}$ size. On sectioning, grey white bony hard and calcified lesion of $3 \times 3 \times 2 \mathrm{~cm}$ was identified. Microscopic examination showed neoplastic lesion composed of oval to pleomorphic cells arranged in nodules and sheets. Nuclei are hyper chromatic to vesicular with variably prominent nucleoli with moderate to marked pleomorphism. Mitotic activity was also noted. Osteoid was present in the lesion. All margins were tumor free. Less than $5 \%$ tumor necrosis noted. Findings were suggestive of osteosarcoma (Figure 3 and 4). Patient was followed routinely. Adjuvant chemotherapy was done. In 2021 he develops chest metastasis and is under treatment.

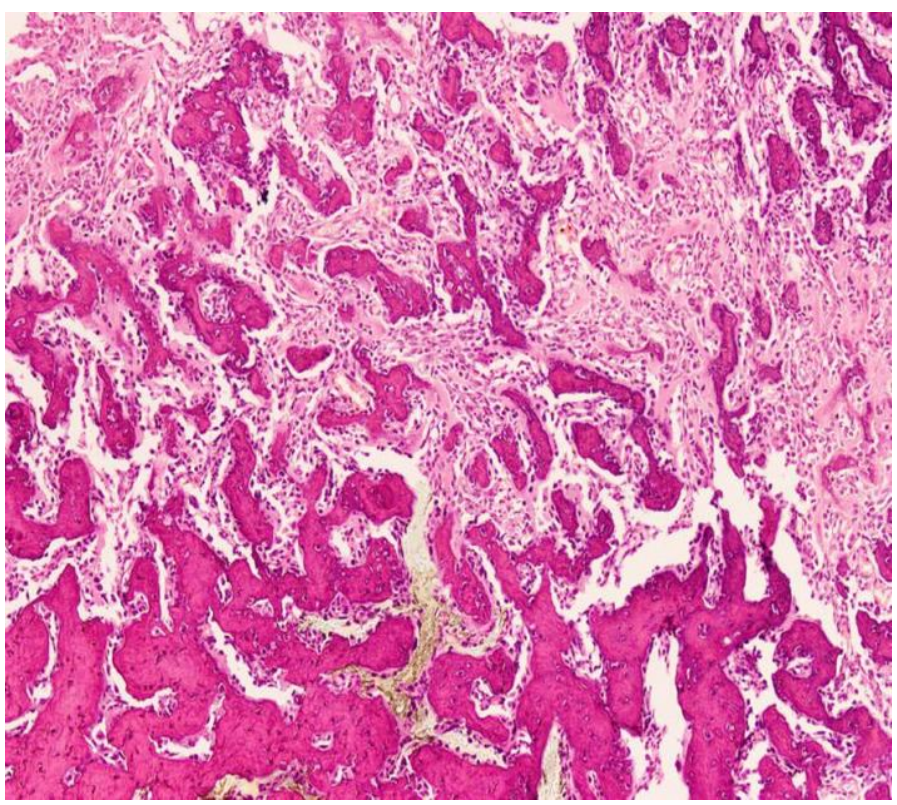

Figure 3: Low power shows an extensive osteoid matrix producing tumor with irregular trabeculae and intervening atypical spindle cells.

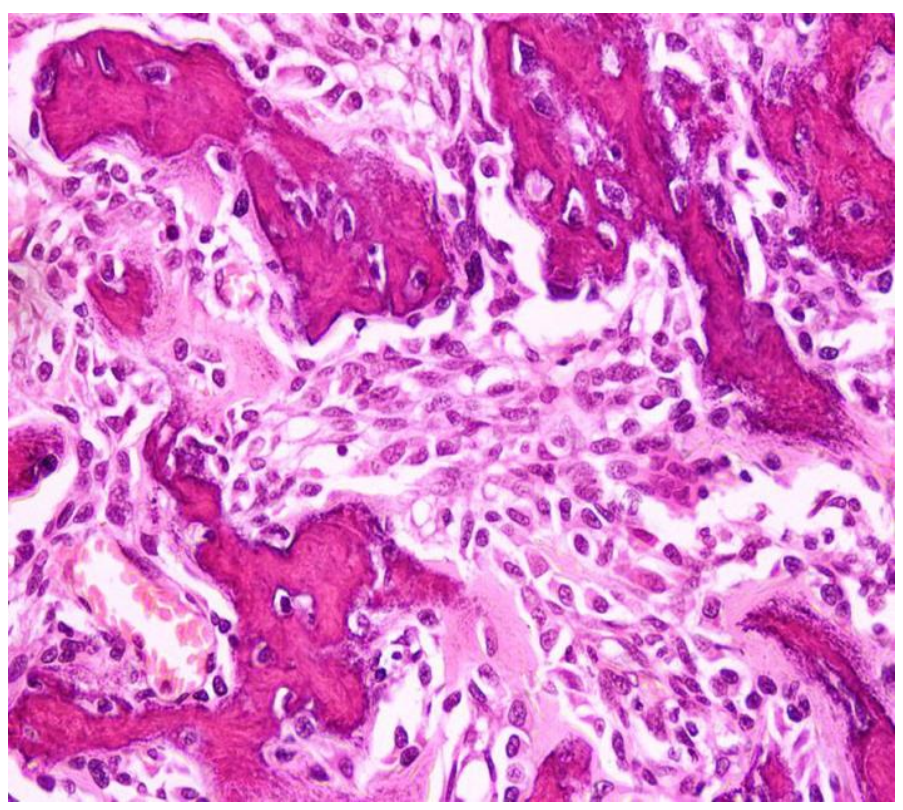

Figure 4: High power shows an extensive osteoid matrix producing tumor with irregular trabeculae and intervening atypical spindle cells 


\section{DISCUSSION}

Owing to the extremely rare nature of extra skeletal osteosarcoma globally, the medical literature has scarcity of publications on this disease. Extra skeletal osteosarcoma is an aggressive, high-grade malignant neoplasm originating from the mesenchyme containing majorly osteoblastic cells [3]. It is most commonly located in the soft tissues of thigh (46\%), succeeded by upper limb (20\%) but can be found in any area of the body [3]. It comprises of $4-5 \%$ of all osteosarcomas and $2 \%$ of all soft tissue sarcomas. It is mostly manifested at the age of $40-70$ years with males being more affected [3]. In the case being discussed here the tumor was grossly identified as a slow growing lump on the right supra scapular region.

The risk is increased with an underlying Paget's disease, fibrous dysplasia or previously performed radio or chemotherapy for other tumors but the patient didn't have any of these known risk factors, however he is hypertensive and taking medications for it [3].

The site of location of the tumor depicts its clinical manifestations. Initially when the tumor is growing, no signs and symptoms are observed. It then takes a rapid turnover, growing into a large size, encroaching onto the nearby nerves, muscle and organs leading to pain and tenderness [5]. The sensory motor movements were intact on the affected side and the tumor growth was restricted to muscles only.

The imaging studies are often nonspecific with mineralized elements in well-defined mass [3]. Magnetic resonance sequences demonstrate a well-circumscribed mass with hemorrhage and enhancing solid components [6]. The same imaging modality was used in our patient as well which showed a right Suprascapular hyper intense mass. Necrosis and hemorrhage in a cystic cavity are common on pathological examination, which in our case was only $5 \%$ [6].

The tumors may grow up to $50 \mathrm{~cm}$ and being firmly attached to the fascia rather than the bone but rarely can be in contact with the periosteum. It is grossly encapsulated and can remain confined to the dermis but sometimes may ulcerate. Microscopically there are anaplastic spindle shaped cells with marked cytological atypia, high mitotic rated with malignant chondroid areas. The neoplastic bone deposition is in a lacy, trabecular pattern [3]. Microscopic examination after a biopsy in this case showed neoplastic lesion composed of oval to pleomorphic cells arranged in nodules and sheets. There are no documented genetic differences between the skeletal and the extra skeletal variant. [3] The differential diagnosis for extra skeletal osteosarcoma includes Myositis ossificans, Synovial and epithelioid sarcoma, Extra skeletal chondrosarcoma, Malignant fibrous histiocytoma, Rhabdomyosarcoma, Hamartoma, Malignant schwannoma, Malignant mesenchymoma, Liposarcoma with metaplastic bone [3].

Because the tumor is itself very rare there is no globally accepted treatment plan and it is widely treated with conventional chemotherapy and surgery. [2][7] However, recent studies have suggested that when extra skeletal osteosarcoma is treated similarly to conventional osteosarcoma the outcomes show promising results. According to different studies the 5-year survival rate in patients who have received chemotherapy for their osteosarcoma varies between $37 \%-66 \%$, which is similar to skeletal osteosarcoma [2][8]. Factors associated with adverse outcomes include distant metastasis, large tumor size and incomplete resection. [8] Globally the current surgical treatment being offered to such patients, include wide margin resection or radical resection and when the tumor involves any extremity amputation is also considered. Chemotherapy, which is mostly doxorubicin based, can be commenced before or after the surgical procedure [2]. Some studies are also in favor of using platinum-based chemotherapy which is associated with low recurrence rates. [7] However, there is no significant difference in the survival rates of patients who have received chemotherapy when compare to those who haven't. Previously it has been reported that tumor size is an important factor, which determines survival [2].

Three cycles of neoadjuvant chemotherapy were done on the patient before undergoing a wide margin resection of the tumor followed by an adjuvant cycle. The tumor was however not adherent to the bone or the overlying skin which gives us more successful results.

\section{CONCLUSION}

Extra skeletal osteosarcoma is an extremely rare, high grade, malignant soft tissue tumor. It can grow into a large size and compress adjacent nerves and vessels leading to pain and tenderness. Regardless of the low incidence rate, knowledge and recognition of the tumor are essential for accurate and timely detection. Our case of extra skeletal osteosarcoma of supra scapular region is a very unique presentation. Wide margin excision along with conventional chemotherapy is the accepted treatment plan.

\section{Conflict of Interest}

We declare that we have no conflict of interest.

\section{Financial Support}

None declared.

\section{REFERENCES}

1. de Azevedo JW, Fernandes TA, Fernandes JV, de Azevedo JC, Lanza DC, Bezerra CM et al. Biology and pathogenesis of human osteosarcoma. Oncology letters. 2020;19(2):1099-116.

2. Nystrom LM, Reimer NB, Reith JD, Scarborough MT, Gibbs CP Jr. The Treatment and Outcomes of Extraskeletal Osteosarcoma: Institutional Experience and Review of The Literature. Iowa Orthop J. 2016;36:98-103.

3. Mavrogenis A. Papagelopoulos P.Soft Tissues: Extraskeletal osteosarcoma. Atlasgeneticsoncology [online] 2013-12 [Cited 2021 Nov 25]; Available at: <http://atlasgeneticsoncology.org/Tumors/ExtraskeletalOsteosar cID5200.html>

4. Mc Auley G, Jagannathan J, O'Regan K, Krajewski KM, Hornick $\mathrm{JL}$, Butrynski J, Ramaiya N. Extraskeletal osteosarcoma: spectrum of imaging findings. American Journal of Roentgenology. 2012;198(1):W31-7.

5. DoveMed.com 2021. Extraskeletal Osteosarcoma. [online] Available at: <https://www.dovemed.com/diseasesconditions/extraskeletal-osteosarcoma/> [Accessed 16 November 2021].

6. Varma DG, Ayala AG, Guo SQ, Moulopoulos LA, Kim EE, Charnsangavej C. MRI of extraskeletal osteosarcoma. Journal of computer assisted tomography. 1993;17(3):414-7.

7. Paludo J, Fritchie K, Haddox CL, Rose PS, Arndt CA, Marks RS et al. Extraskeletal osteosarcoma: outcomes and the role of chemotherapy. American journal of clinical oncology. 2018:41(9):832-37.

8. Narayanan ST, Gopalakrishnan MK, Ibrahim SS, Sankar R. Extraskeletal osteosarcoma-a case report. Journal of clinical and diagnostic research: JCDR. 2016;10(1):ED03-04. 